Acta Crystallographica Section B

Structural

Science

ISSN 0108-7681

Editor: Carolyn P. Brock

\title{
Neutron diffraction investigation of the temperature dependence of crystal structure and thermal motions of red $\mathbf{H g I}_{2}$
}

Dieter Schwarzenbach, Henrik Birkedal, Marc Hostettler and Peter Fischer

Copyright $\odot$ International Union of Crystallography

Author(s) of this paper may load this reprint on their own web site or institutional repository provided that this cover page is retained. Republication of this article or its storage in electronic databases other than as specified above is not permitted without prior permission in writing from the IUCr.

For further information see http://journals.iucr.org/services/authorrights.html 
Acta Crystallographica Section B

Structural

Science

ISSN 0108-7681

\section{Neutron diffraction investigation of the tempera- ture dependence of crystal structure and thermal motions of red $\mathrm{HgI}_{2}$}

\section{Dieter Schwarzenbach, ${ }^{\mathrm{a} *}$ Henrik Birkedal, ${ }^{ } \neq$Marc Hostettler ${ }^{\mathrm{a}} \S$ and Peter Fischer ${ }^{b}$}

\author{
aLaboratory of Crystallography, Ecole Polytech- \\ nique Fédérale, Le Cubotron, $\mathrm{CH}-1015$ \\ Lausanne, Switzerland, and baboratory for \\ Neutron Scattering, ETH Zürich and Paul \\ Scherrer Institute, $\mathrm{CH}-5232$ Villigen PSI, Swit- \\ zerland
}

\author{
₹ Present address: Department of Chemistry, \\ University of Aarhus, DK-8000 Aarhus C, \\ Denmark. \\ $\S$ Present address: chemin de la Coudrette 35, \\ $\mathrm{CH}-1012$ Lausanne, Switzerland.
}

Correspondence e-mail:

dieter.schwarzenbach@epfl.ch
The structure of, and anisotropic thermal motions in, the red semiconductor tetrahedral layer structure of $\mathrm{HgI}_{2}$ have been studied with neutron powder diffraction as a function of temperature from 10 to $293 \mathrm{~K}$. Average thermal displacement parameters $U_{\text {eq }}$ of the two atoms are comparable in size at $10 \mathrm{~K}$, but $U_{\text {eq }}(\mathrm{Hg})$ increases considerably faster with temperature than $U_{\text {eq }}(\mathrm{I})$, the $\mathrm{Hg}-\mathrm{I}$ bond being highly non-rigid. The anisotropic displacement tensor $\boldsymbol{U}(\mathrm{I})$ is strongly anisotropic with one term about twice as large as the others, while $\boldsymbol{U}(\mathrm{Hg})$ is nearly isotropic. All displacement tensor elements, except $U_{22}(\mathrm{I})$, increase faster with temperature than harmonic quantum oscillator curves indicating a softening of the isolated-atom potentials at large amplitudes. A lattice dynamical model provides arguments that the anisotropic thermal motions of I are dominated by a soft mode with a wavevector at the $\left[\begin{array}{lll}\frac{1}{2} & \frac{1}{2} & 0\end{array}\right]$ boundary of the Brillouin zone consisting essentially of coupled librations of the $\mathrm{HgI}_{4}$ tetrahedra, and by translations of the entire layer. The large vibration amplitudes of $\mathrm{Hg}$ suggest weak $\mathrm{Hg}-\mathrm{I}$ force constants compared with the I-I force constants, allowing $\mathrm{Hg}$ to move quite freely inside the tetrahedra. The libration mode induces dynamic deformations of the $\mathrm{Hg}-\mathrm{I}$ bond with twice its frequency. This provides a mechanism for the anharmonicity and may explain the lightening of the color from red to orange upon cooling at $c a 80 \mathrm{~K}$.

\section{Introduction}

Mercuric iodide, $\mathrm{HgI}_{2}$, forms many different structures depending on temperature and pressure. At ambient conditions, it also shows several metastable structures co-existing with the stable red form, phase III (Jeffrey \& Vlasse, 1967). Several of these structures have been determined in detail (Hostettler et al., 2001). The latest version of the phase diagram has been published by Hostettler \& Schwarzenbach (2005). The red phase III adopts a tetrahedral semiconductor structure (Jeffrey \& Vlasse, 1967; Hostettler \& Schwarzenbach, 2005), where the I atoms form a cubic closest packing and the $\mathrm{Hg}$ atoms occupy layers of corner-linked tetrahedral voids with all $\mathrm{Hg}-\mathrm{I}-\mathrm{Hg}$ and $\mathrm{I}-\mathrm{Hg}-\mathrm{I}$ angles close to tetrahedral (Fig. 1). This form is of technological interest because of its opto-electronic properties (Bube, 1957). It is currently used in $\gamma$ - and X-ray detectors (Gospodinov et al., 2005). At ambient pressure, this structure is stable from the lowest temperatures to $c a 400 \mathrm{~K}$, at which temperature a firstorder phase transition to a yellow phase II occurs, exhibiting $\mathrm{HgI}_{2}$ molecules (Hostettler et al., 2003). Differential thermal analysis data (Tonkov \& Tikhomirova, 1971) indicate the existence of a phase transition at moderate temperatures and pressures of phase III into phase IV, whose structure is iden-
Received 7 June 2007

Accepted 4 September 2007 
tical to that of phase III. The III-IV transformation is not associated with a change of the volume of the unit cell. However, it shows a change of 'tetragonality', with $\tau=c / a-$ $8^{1 / 2}$ changing sign from positive to negative. At $\tau=0$, the metric of the iodine sublattice is cubic with $\left|\mathbf{a}_{1} \pm \mathbf{a}_{2}\right|=2^{1 / 2} a=$ $c / 2$. The III-IV phase boundary has been associated with an anti-isostructural transition, and has been tentatively extrapolated to ambient pressure and $c a 80 \mathrm{~K}$ (Hostettler \& Schwarzenbach, 2005), where $\tau \simeq 0$. The red color fades with decreasing temperature. The compound is orange at $80 \mathrm{~K}$.

The thermal displacement parameters of the red phase III have the peculiar property that they are larger for $\mathrm{Hg}$ than for $\mathrm{I}$, at least in the isotropic approximation, in spite of $\mathrm{Hg}$ being much heavier than I. The study of the anisotropic displacement parameters as functions of temperature may yield an explanation of this observation and give further insight into the elusive anti-isostructural transition III-IV. Precise X-ray studies of $\mathrm{HgI}_{2}$ are hampered by the following obstacles:

(1) Absorption is strong even for short-wavelength radiation and absorption corrections require precise measurements of crystal shapes and diffraction geometries.

(2) The use of graphite-monochromated radiation in singlecrystal studies always results in scan-truncation errors (Rousseau et al., 2000; Lenstra et al., 2001), in particular for the high-order reflections which are indispensible for a precise determination of displacement parameters.

(3) $\mathrm{HgI}_{2}$ crystals lose substance by sublimation at room temperature and in open gas-flow refrigerators. Therefore, for time-consuming diffraction studies crystals have to be protected.

In contrast, neutron powder diffraction offers advantages in the determination of thermal motion parameters. Absorption effects in $\mathrm{HgI}_{2}$ are small. The wavelength dispersion in a monochromated neutron beam is much better defined than in a graphite-monochromated X-ray beam; it is well known that displacement parameters measured with neutrons are in general more trustworthy, while those from graphite-mono-

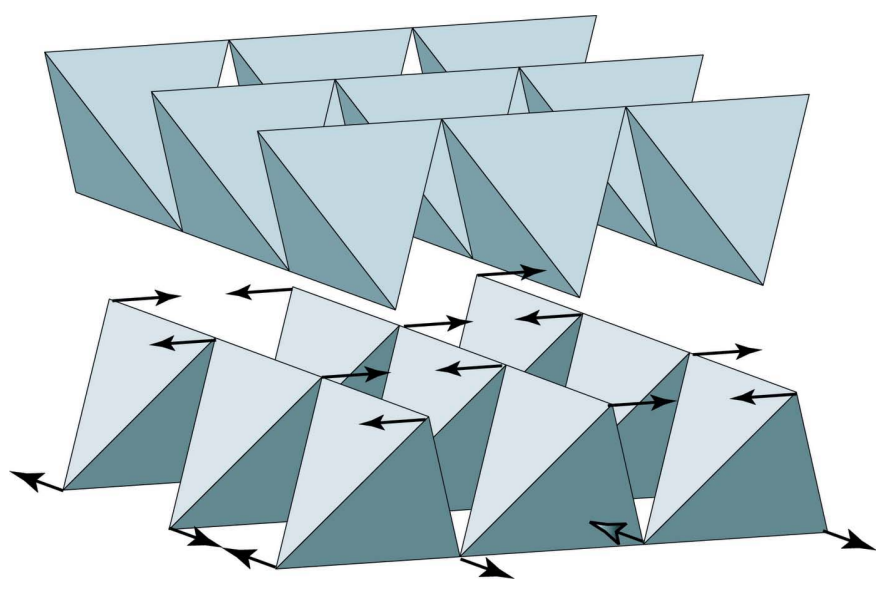

Figure 1

Structure of red $\mathrm{HgI}_{2}$ (program ATOMS; Dowty, 2006). The $\mathrm{HgI}_{4}$ tetrahedra are corner-linked into layers which are stacked along $\mathbf{c}$. The arrows are along $\mathbf{a}_{1}$ and $\mathbf{a}_{2}$ of the tetragonal unit cell and indicate the dominant thermal vibration mode of $\mathrm{I}$. chromated X-rays are most often too large (Lenstra et al., 2001). The sample can be enclosed in a solid container that is transparent to neutrons. For a simple structure such as $\mathrm{HgI}_{2}$, powder diffraction can yield anisotropic displacement parameters and it is an excellent method for calibrating results obtained by X-ray diffraction.

In this work, we present anisotropic thermal displacement parameters in $\mathrm{HgI}_{2}$ as functions of temperature from $10 \mathrm{~K}$ to room temperature determined from powder neutron diffraction, as well as results obtained at room temperature from single-crystal X-ray diffraction. Preliminary results have been published in Hostettler et al. (2001). We now propose a lattice dynamical model explaining the anisotropy of the displacement tensor of I, and a mechanism for the anharmonicity.

\section{Experimental}

For neutron diffraction, commercial red $\mathrm{HgI}_{2}$ powder (Fluka 83379) was enclosed under He gas atmosphere in a cylindrical $\mathrm{V}$ container of $8 \mathrm{~mm}$ diameter and height $52 \mathrm{~mm}$. The powder diffraction measurements were performed on the high-resolution multidetector powder diffractometer HRPT for thermal

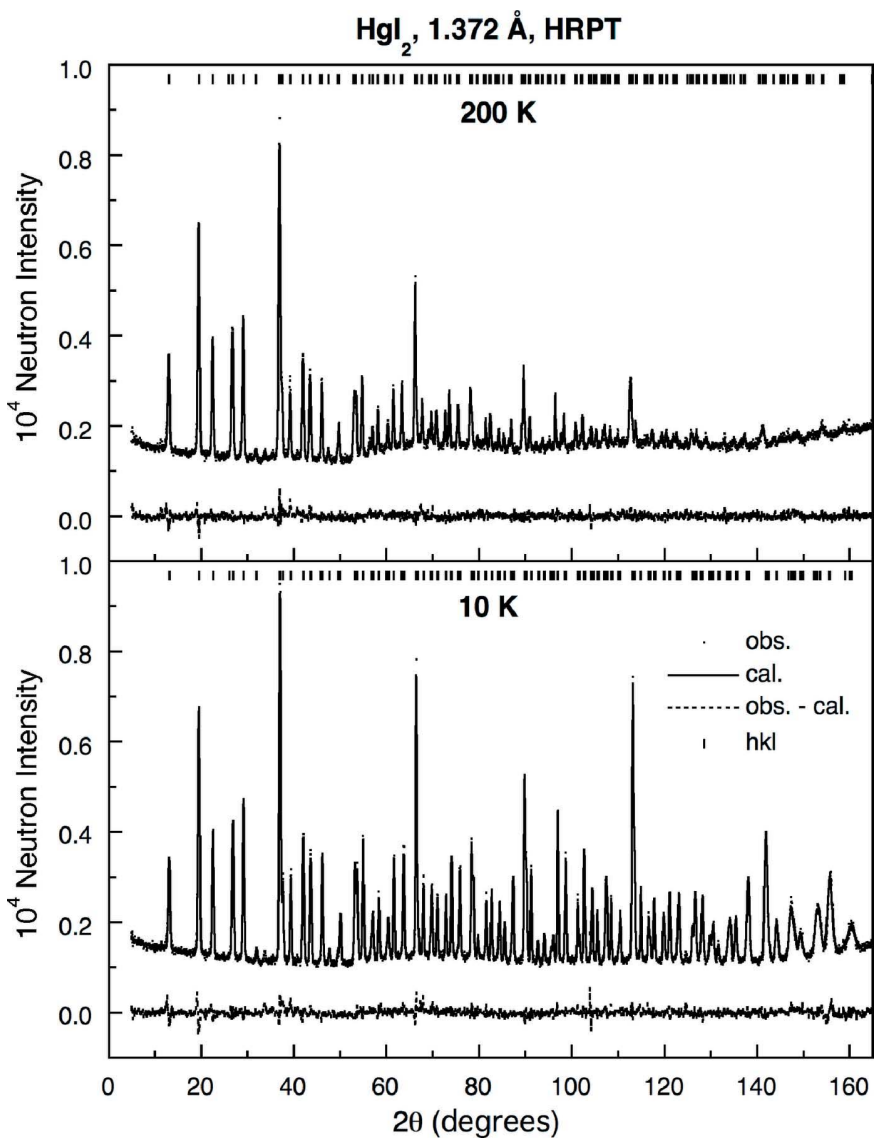

Figure 2

Observed (points), calculated (line) and difference (obs. - calc., dashed line) neutron diffraction patterns of $\mathrm{HgI}_{2}$ at $200 \mathrm{~K}$ (anisotropic displacement factors) and at $10 \mathrm{~K}$ (isotropic displacement factors), respectively. Note at $200 \mathrm{~K}$ the pronounced decrease of the Bragg intensities at high scattering angles as well as the presence of significant diffuse intensity. 


\section{research papers}

\section{Table 1}

Lattice parameters $a$ and $c$ of red $\mathrm{HgI}_{2}$ as functions of the temperature $T$, refined from the powder neutron intensities, and single-crystal X-ray data, with anisotropic displacement parameters.

Tetragonal, space group $P 4_{2} / n m c$ (No. 137), origin at $\overline{1}$; $\mathrm{Hg}$ in position $2(a), 1 / 43 / 41 / 4$, site symmetry $\overline{4} m 2$; I in position $4(d), 1 / 41 / 4 z$, site symmetry $2 m m$. The agreement values $R_{\mathrm{wp}}, R_{\exp }, \chi^{2}$ and $R_{\mathrm{B}}$ concern weighted profile intensities, a statistically expected value, goodness-of-fit and integrated intensities (Rodriguez-Carvajal, 1993). Standard uncertainties are given within parentheses and correspond to the last relevant digit.

\begin{tabular}{llllllll}
\hline$T(\mathrm{~K})$ & $a(\AA)$ & $c(\AA)$ & $z(\mathrm{I})$ & $R_{\text {wp }}$ & $R_{\text {exp }}$ & $\chi^{2}$ & $R_{\mathrm{B}}$ \\
\hline 10 & $4.358(1)$ & $12.300(3)$ & $0.3915(1)$ & 0.041 & 0.025 & 2.8 & 0.034 \\
30 & $4.358(1)$ & $12.306(3)$ & $0.3914(2)$ & 0.064 & 0.027 & 5.5 & 0.035 \\
60 & $4.359(1)$ & $12.319(3)$ & $0.3912(1)$ & 0.035 & 0.023 & 2.5 & 0.039 \\
100 & $4.360(1)$ & $12.337(3)$ & $0.3909(2)$ & 0.039 & 0.023 & 2.9 & 0.050 \\
200 & $4.365(1)$ & $12.389(3)$ & $0.3904(2)$ & 0.037 & 0.023 & 2.7 & 0.072 \\
293 & $4.370(1)$ & $12.437(3)$ & $0.3898(6)$ & 0.072 & 0.033 & 4.8 & 0.086 \\
$293 X$ & $4.3675(6)$ & $12.470(3)$ & $0.3890(2)$ & - & - & 4.9 & 0.086 \\
\hline
\end{tabular}

\section{Table 2}

$\mathrm{Hg}-\mathrm{I}$ distances $(\AA)$, apical $\mathrm{I}-\mathrm{Hg}-\mathrm{I}$ angles $\left(^{\circ}\right), \mathrm{I}-\mathrm{I}$ interlayer contact distances $(\AA)$, r.m.s. libration amplitudes $\left(L_{33}\right)^{1 / 2}$ of $\mathrm{HgI}_{4}$ tetrahedra about $\mathbf{c}\left(^{\circ}\right)$ as functions of the temperature $T$.

The equatorial $\mathrm{I}-\mathrm{Hg}-\mathrm{I}$ angles are obtained from $\cos ($ apical $)+2 \cos$ (equatorial $)=-1$. The apical angles are bisected by the $\mathbf{c}$ direction, the equatorial angles are bisected by the $\mathbf{a}_{1}$ and $\mathbf{a}_{2}$ directions. The $\mathrm{Hg}-\mathrm{I}-\mathrm{Hg}$ angles are the same as the apical $\mathrm{I}-\mathrm{Hg}-\mathrm{I}$ angles.

\begin{tabular}{lllll}
\hline$T$ & $d(\mathrm{Hg}-\mathrm{I})$ & $\alpha(\mathrm{I}-\mathrm{Hg}-\mathrm{I})$ & $d(\mathrm{I}-\mathrm{I})$ & $\left(L_{33}\right)^{1 / 2}$ \\
\hline 10 & $2.789(1)$ & $102.77(5)$ & $4.077(1)$ & $1.3(1)$ \\
30 & $2.789(1)$ & $102.76(8)$ & $4.079(1)$ & $1.6(1)$ \\
60 & $2.788(1)$ & $102.81(6)$ & $4.085(1)$ & $1.8(1)$ \\
100 & $2.788(1)$ & $102.89(7)$ & $4.088(1)$ & $2.3(1)$ \\
200 & $2.791(2)$ & $102.90(9)$ & $4.111(2)$ & $3.7(1)$ \\
293 & $2.792(4)$ & $103.0(3)$ & $4.131(4)$ & $4.6(2)$ \\
\hline
\end{tabular}

neutrons (Fischer et al., 2000), situated at the continuous spallation neutron source SINQ (Fischer, 1997) of Paul Scherrer Institute (PSI) at Villigen, Switzerland. A primary Soller collimation of $12 \mathrm{~min}$, secondary collimation of $24 \mathrm{~min}$ and scattering angle step of $0.05^{\circ}$ were used. The neutron wavelength, $\lambda=1.3717$ (3) $\AA$, calibrated with an Si standard sample, was obtained from a wafer-type, vertically focusing $\mathrm{Ge}$ monochromator. The sample was cooled by means of a closedcycle He refrigerator. Data were collected at 10, 30, 60, 100, 200 and $293 \mathrm{~K}$.

The profile data were analysed with a recent version of the FULLPROF program package, based on the internal neutron scattering amplitues (Rodriguez-Carvajal, 1993). The data were corrected for absorption; the product of the linear absorption coefficient and the cylinder radius $\mu r=0.378$ was obtained from the measured neutron transmission. The background was well described by 12 cosine Fourier parameters (Rodriguez-Carvajal, 1993). Anisotropic thermal displacement parameters were refined for all temperatures and compared with the corresponding isotropic refinements. Refinements converged easily, and agreement between the isotropic and anisotropic model parameters was excellent. In particular, the equivalent isotropic displacement parameters $U_{\text {eq }}=\left(U_{11}+U_{22}+U_{33}\right) / 3$ from the anisotropic tensors agreed closely with the isotropic values. The isotropic results therefore need not be reported here. In total, 28 parameters (or 25 for the isotropic refinements) were refined against approximately 230 Bragg reflections $(h k l)$ contributing to the measured neutron diffraction patterns. Fig. 2 shows two characteristic diffraction patterns. Obviously, the amplitudes of atomic thermal motions are quite large already at $200 \mathrm{~K}$, as evidenced by the strong decrease of Bragg intensities at higher scattering angles. There are also significant diffuse intensities visible in the neutron diffraction patterns.

Single-crystal X-ray data were measured on a Stoe image-plate diffractometer with Mo $K \alpha$ radiation, $\lambda=0.71073 \AA$. The crystal was obtained by recrystallization from 2-chloroethanol. The crystal shape was measured with a telescope and used for absorption correction by numerical integration. Data collection at $293 \mathrm{~K}$ consisted of 200 images taken with an oscillation angle of $1^{\circ}$ and an exposition time of $300 \mathrm{~s}$ per image. The structure was refined anisotropically by standard least-squares against the structure factors squared $|F|^{2}$ (Hostettler, 2002).

\section{Analysis of geometrical properties}

Table 1 reports the lattice constants and the atomic coordinate $z$ of I as functions of temperature and the agreement values of the refinements. Table 2 shows intra- and interlayer distances and angles, and Table 3 shows the thermal displacement parameters, i.e. the mean-square displacements (m.s.d.) in $\AA^{2}$. Figs. 3 and 4 show the corresponding graphical representations of these results. The structure is clearly the same in the whole temperature range from $10 \mathrm{~K}$ to room temperature. The unitcell volume and the lattice constant $c$ increase linearly above $100 \mathrm{~K}$. In contrast, $a$ expands less than $c$ at low temperatures,

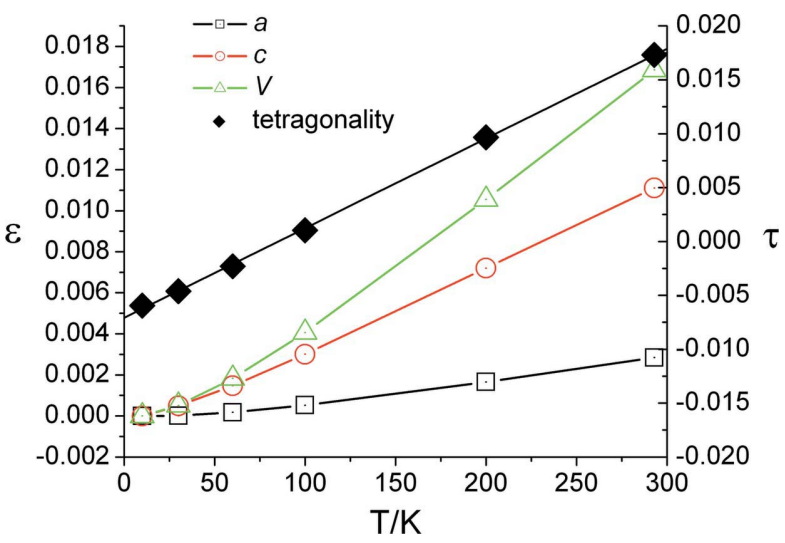

Figure 3

Temperature dependence of lattice constants and unit-cell volume of red $\mathrm{HgI}_{2} . \varepsilon(T)$ is the deformation $[X(T)-X(T=10 \mathrm{~K})] / X(T=10 \mathrm{~K})$ with $X=a, c$ and $V ; \tau(T)=c(T) / a(T)-8^{1 / 2}$ is the tetragonality $(\tau=0$ for a metrically cubic iodine sublattice). 
Table 3

Thermal displacement tensors of red $\mathrm{HgI}_{2}$ as functions of the temperature $T$, refined from the powder neutron intensities and single-crystal X-ray data.

For $\mathrm{Hg}, U_{11}=U_{22}, U_{33}, U_{12}=U_{13}=U_{23}=0$; for $\mathrm{I}, U_{11}, U_{22}, U_{33}, U_{12}=U_{13}=U_{23}=0$. The temperature factor expression is $\exp \left[-2 \pi^{2}\left(U_{11} h^{2}+U_{22} k^{2}\right) / a^{2}+U_{33} l^{2} / c^{2}\right]$, the equivalent isotropic expression is $\exp \left[-8 \pi^{2} U_{\text {eq }}(\sin \theta / \lambda)^{2}\right], U_{\text {eq }}=\left(U_{11}+U_{22}+U_{33}\right) / 3$. Standard uncertainties are given within parentheses and correspond to the last relevant digit.

\begin{tabular}{llllllll}
\hline$T$ & $U_{\text {eq }} \mathrm{Hg}$ & $U_{\text {eq }} \mathrm{I}$ & $U_{11} \mathrm{Hg}$ & $U_{33} \mathrm{Hg}$ & $U_{11} \mathrm{I}$ & $U_{22} \mathrm{I}$ & $U_{33} \mathrm{I}$ \\
\hline 10 & $0.0013(2)$ & $0.0011(5)$ & $0.0013(2)$ & $0.0011(2)$ & $0.0024(5)$ & $0.0001(5)$ & $0.0008(5)$ \\
30 & $0.0035(3)$ & $0.0027(8)$ & $0.0036(3)$ & $0.0032(5)$ & $0.0048(9)$ & $0.0009(9)$ & $0.0025(8)$ \\
60 & $0.0071(2)$ & $0.0057(6)$ & $0.0071(2)$ & $0.0071(3)$ & $0.0088(6)$ & $0.0040(6)$ & $0.0043(5)$ \\
100 & $0.0122(3)$ & $0.0103(8)$ & $0.0124(3)$ & $0.0120(5)$ & $0.0157(9)$ & $0.0078(8)$ & $0.0074(7)$ \\
200 & $0.0272(5)$ & $0.022(1)$ & $0.0276(5)$ & $0.0264(7)$ & $0.034(1)$ & $0.015(1)$ & $0.016(1)$ \\
293 & $0.042(2)$ & $0.033(5)$ & $0.042(2)$ & $0.042(3)$ & $0.052(5)$ & $0.022(4)$ & $0.026(5)$ \\
$293 X$ & $0.053(3)$ & $0.043(3)$ & $0.050(2)$ & $0.059(3)$ & $0.057(2)$ & $0.031(2)$ & $0.042(3)$ \\
$293 X-\Delta U$ & $0.042(3)$ & $0.033(3)$ & $0.040(2)$ & $0.049(3)$ & $0.047(2)$ & $0.021(2)$ & $0.032(3)$ \\
\hline
\end{tabular}

a straight line passing through the origin at $T=0$. However, all observed $U$ s, with the exception of $U_{22}(\mathrm{I})$, increase faster than harmonic oscillator curves. This indicates anharmonic motions corresponding to a softening at large displacement amplitudes of the isolated-atom potentials, compared with the parabolic harmonic potentials. Anharmonic effects can be taken into account in (1) through a quasi-harmonic Grüneisentype temperature dependence of the frequency $\omega$ (Bürgi et al., 2000)

$$
\omega(T)=\omega_{0}\left[1-\gamma\left(V-V_{\min }\right) / V_{\min }\right],
$$

where $\gamma$ is a Grüneisen constant, $V$ is the unit-cell volume and $V_{\min }$ the cell and the increase is not yet linear at $100 \mathrm{~K}$. Thermal expansion in the covalently bonded $\mathrm{HgI}_{2}$ layers is small over the whole temperature range. The shape of the $\mathrm{HgI}_{4}$ tetrahedron hardly changes and there is virtually no expansion of the $\mathrm{Hg}-\mathrm{I}$ distance (Table 2). The thermal expansion of the unit cell is dominated by the expansion of the interlayer I-I contact distance (Table 2), which is much larger than the expansion of the intralayer I-I distance [the I-I distances corresponding to the equatorial $\mathrm{I}-\mathrm{Hg}-\mathrm{I}$ angles expand from 4.649 (2) $\AA$ at $10 \mathrm{~K}$ to 4.66 (1) $\AA$ at $293 \mathrm{~K}$ ]. The geometry of the structure does not show evidence of the elusive III-IV phase transition. The tetragonality depends linearly on temperature (correlation coefficient 0.99978 ) and changes sign at $85 \mathrm{~K}$.

\section{Analysis of the thermal displacement parameters}

\subsection{Quasi-harmonic quantum oscillator curves}

Fig. 4 shows the displacement parameters of Table 3 as functions of temperature. At $10 \mathrm{~K}$, the $U$ values, i.e. the meansquare displacements (m.s.d.), of $\mathrm{Hg}$ and I are close to the zero-point motions, but they increase strongly with temperature. The thermal motion of $\mathrm{Hg}$ is nearly isotropic, whereas that of $\mathrm{I}$ is highly anisotropic. $U_{11}(\mathrm{Hg})$ and $U_{33}(\mathrm{Hg})$ are consistently, and at higher temperatures, considerably larger than $U_{22}(\mathrm{I}), U_{33}(\mathrm{I})$ and $U_{11}(\mathrm{I})-U_{22}(\mathrm{I})$, although the ratio of the atomic masses $M(\mathrm{Hg}) / M(\mathrm{I})$ is $c a$ 1.6. The r.m.s. displacements along the $\mathrm{Hg}-\mathrm{I}$ bonds are $u_{\text {bond }}=\left\{U_{22} \sin ^{2}(\alpha / 2)\right.$ $\left.+U_{33}\left[1-\sin ^{2}(\alpha / 2)\right]\right\}^{1 / 2}$, where $\alpha$ is the $\mathrm{I}-\mathrm{Hg}-\mathrm{I}$ angle of Table 2. The difference $u_{\text {bond }}(\mathrm{Hg})-u_{\text {bond }}(\mathrm{I})$ increases from $0.015 \AA$ at $10 \mathrm{~K}$ to $0.052 \AA$ at $293 \mathrm{~K}$, indicating the $\mathrm{Hg}-\mathrm{I}$ bond to be highly non-rigid. The curves in Fig. 4 also indicate the displacements to be anharmonic. The temperature dependence of harmonic thermal motions ought to obey a Debye curve or a harmonic oscillator curve (Bürgi et al., 2000)

$$
U(T)=[\hbar /(2 \omega m)] \operatorname{coth}\left[\hbar \omega /\left(2 k_{\mathrm{B}} T\right)\right],
$$

where $\hbar$ is Planck's constant, $\omega$ the vibrational circular frequency, $m$ the mass of the oscillator and $k_{\mathrm{B}}$ Boltzmann's constant. In the high-temperature limit, this curve approaches volume at the lowest temperature of measurement $(10 \mathrm{~K})$. The thermal expansion of the unit cell is represented by $(V-$ $\left.V_{\text {min }}\right) / V_{\text {min }}=b_{1} T+b_{2} T^{2}$, where the coefficients have been obtained by a fit to the data of Table 1 . Fitting (1) and (2) to the data of Table 3 gives: $\gamma=5.4$ (2.0), 6.9 (1.8), 0 (fixed), 8.7 (2.7), $7.6(1.7)$ and $\omega_{0} / 2 \pi c=31(1), 32(1), 50.2(1), 54(3), 49$ (1) $\mathrm{cm}^{-1}$ for $U_{11}(\mathrm{Hg}), U_{33}(\mathrm{Hg}), U_{22}(\mathrm{I}), U_{33}(\mathrm{I}), U_{11}(\mathrm{I})-U_{22}(\mathrm{I})$, respectively; $c$ is the speed of light, uncertainties are given in parentheses, for the choice of $U_{11}(\mathrm{I})-U_{22}(\mathrm{I})$ see (4). The curves in Fig. 4 show the quality of the fit to be worst at the lowest temperatures. The frequencies are of the order of those reported by Prevot et al. (1978) and Biellmann \& Prevot (1980). Also refining $\gamma$ for $U_{22}(\mathrm{I})$ gives $\gamma$ $=4.5(6.0)$ and a frequency of $54(5) \mathrm{cm}^{-1}$, but no superior fit.

The refined anisotropic displacement parameters from $\mathrm{X}$ ray data are considerably larger than those from neutron data. This may be explained by scan truncation errors in the X-ray data which may be even larger than postulated in Lenstra et al. (2001) owing to the automatic integration procedure of the

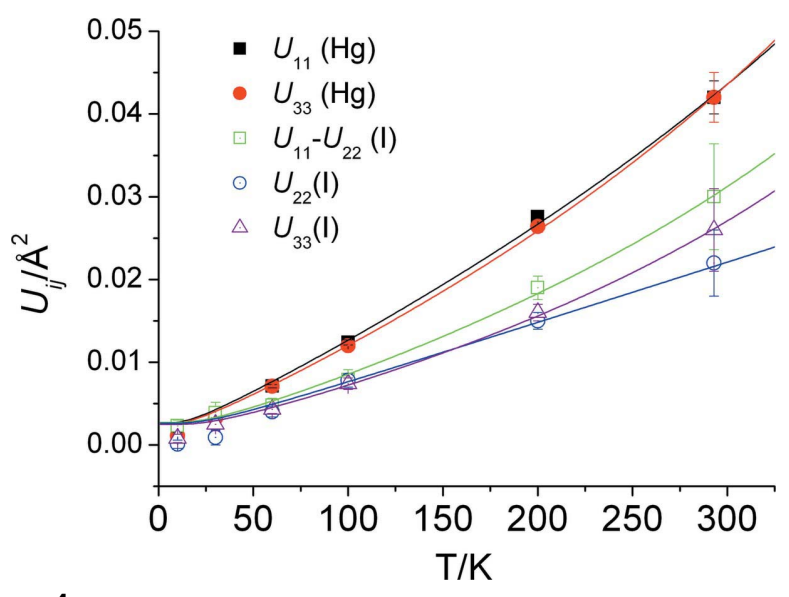

Figure 4

Temperature dependence of the anisotropic displacement parameters in $\mathrm{HgI}_{2}$. The quasi-harmonic quantum oscillator curves have been obtained using equations (1) and (2) by adjusting a Grüneisen constant $\gamma$ and a frequency $\omega_{0}$ to the observations. For $U_{22}(\mathrm{I})$, the harmonic curve $(\gamma=0)$ is shown. $U_{11}(\mathrm{I})-U_{22}(\mathrm{I})$ is reported, rather than $U_{11}(\mathrm{I})$, because it represents the librational eigenmode, see equation (4). 


\section{research papers}

image-plate data. By subtracting a correction factor, $U(\mathrm{X}$ ray,corr $)=U(\mathrm{X}$-ray $)-0.01$, a reasonable agreement with the neutron data is achieved (Table 3). In particular, $U_{11}(\mathrm{I})-$ $U_{22}(\mathrm{I})$, to be discussed below, agrees closely.

\subsection{Phonon model}

The thermal motion of I is dominated by the tensor element $U_{11}$, while $U_{22}$ is small and increases only moderately with temperature. The corresponding motions are, respectively, perpendicular and parallel to the densest rows of I atoms, i.e. the straight lines of corner-linked tetrahedral edges. More than anything else, this suggests coupled librations of tetrahedra about the $c$ axis normal to the $\mathrm{HgI}_{2}$ layers (Fig. 1), adjacent tetrahedra librating in opposite senses. To support this interpretation, we show in the following that this movement is indeed a phonon mode with particularly low frequency and thus high amplitude. Phonon dispersion curves for wavevectors along the $a$ axis [100] have been measured by Prevot et al. (1978) and calculated theoretically by Sim et al. (1994). The modes at the Brillouin zone center have been studied with IR (Biellmann \& Prevot, 1980) and Raman (Karmakar et al., 2005) spectroscopies. However, the wavevector of the mode we are interested in belongs to the boundary point $M$ of the Brillouin zone and has the [110] direction. We therefore present a simple two-dimensional model for which phonon dispersion curves can easily be calculated as functions of a set of force constants, representing qualitatively some of the dispersion curves for an isolated $\mathrm{HgI}_{2}$ layer. We then show that for the librational mode we are interested in, the solution for this simple model closely approximates a degenerate soft mode of the complete $\mathrm{HgI}_{2}$ crystal structure. We also attempt to explain the observation that $U_{\text {eq }}(\mathrm{Hg})>U_{\text {eq }}(\mathrm{I})$. Details of the force constant model and types of eigenvectors have been deposited. ${ }^{1}$

4.2.1. Vibrations of a chessboard. The unit cell of an isolated $\mathrm{HgI}_{2}$ layer contains three atoms, one $\mathrm{Hg}$ and two $\mathrm{I}$. Wavevectors are of the type $\mathbf{q}=2 \pi\left(q_{1} \mathbf{a}_{1} *+q_{2} \mathbf{a}_{2} *\right),|\mathbf{q}|=2 \pi / \lambda$ ( $\Sigma$ direction). We assume the following four types of forceconstant tensors for nearest-neighbor interactions: $\mathrm{Hg}-\mathrm{I}$ from the center to the corners of $\mathrm{agI}_{4}$ tetrahedron, two types of strong I-I interactions along the edges of a tetrahedron parallel (apical) and inclined (equatorial) to the plane of the layer, and a weak I-I interaction between different tetrahedra, which is of the same length as the apical tetrahedral edge. This choice of interactions is very different from that of Sim et al. (1994) who include $\mathrm{Hg}-\mathrm{Hg}$ interactions, but do not assign interactions along the four equatorial tetrahedral edges. This omission corresponds to $\alpha_{1}=\alpha_{2}=0$ in (3) below and gives accidental degeneracies at point $M$. Following the method of calculation of Willis \& Pryor (1975), a $9 \times 9$ dynamical matrix may be derived, some of whose elements are complex. The corresponding phonon dispersion curves are in general not easily obtained in a closed form and require numerical

\footnotetext{
${ }^{1}$ Supplementary data for this paper are available from the IUCr electronic archives (Reference: BK5060). Services for accessing these data are described at the back of the journal.
}

calculations for each wavevector of interest. We therefore devise a simpler model whose dispersion curves can be calculated in a closed form and show the features of the vibrations of a $\mathrm{HgI}_{2}$ layer of interest to us. We consider waves in an isolated two-dimensional layer built only of iodine where filled and empty tetrahedra are distinguished by the choice of force constants. We also consider only atomic displacements parallel to the plane of the layer. Vibrations of this model thus do not comprise waves with polarization components along $\mathbf{c}$, nor movements of $\mathrm{Hg}$ against I. The corresponding dispersion curves are thus only approximately those of a complete threedimensional layer, but they can be calculated explicitly. Since the $z$ coordinates of the atoms do not play any role, the problem reduces to the calculation of the vibrations of the projection of the layer onto its plane which is a chessboard, with the black squares representing filled tetrahedra and the white squares empty tetrahedra. There are two translationally non-equivalent atoms per unit cell, resulting in four phonon branches, two acoustical and two optical. When the origin of the coordinate system is placed in the center of a black square, the atom coordinates are $\frac{1}{2}, 0$ and $0, \frac{1}{2}$ for atoms 1 and 2, respectively. Each atom interacts with eight neighbors, four along the edges of the squares $\mathbf{a}_{1} \pm \mathbf{a}_{2}$ (tensor $\boldsymbol{\alpha}$ ), two along the diagonals of the black squares (tensor $\boldsymbol{\beta}$ ), and two along the diagonals of the white squares (tensor $\gamma$ ). Symmetry restricts each interaction to two force constants, one along the bond (longitudinal length deformation, index 1) and one perpendicular to the bond (transverse deformation, index 2). There are thus six force constants: $\alpha_{1}, \alpha_{2}, \beta_{1}, \beta_{2}, \gamma_{1}, \gamma_{2}$. The $\alpha$ and $\beta$ constants represent forces in a relatively rigid tetrahedron occupied by $\mathrm{Hg}$ and are expected to be large, whereas $\gamma$ represents the non-bonded interactions between different filled tetrahedra and is expected to be small. It is a student's exercise (Willis \& Pryor, 1975) to work out the $4 \times 4$ dynamical matrix as a function of the wavevector components $\left(q_{1}\right.$, $q_{2}$ ). The librational mode proposed in Fig. 1 belongs to the wavevector along $\mathbf{a}_{1} \pm \mathbf{a}_{2}$, with components $q_{1}=q_{2}=q$ (specifically point $M$ on the Brillouin zone boundary with $q=$

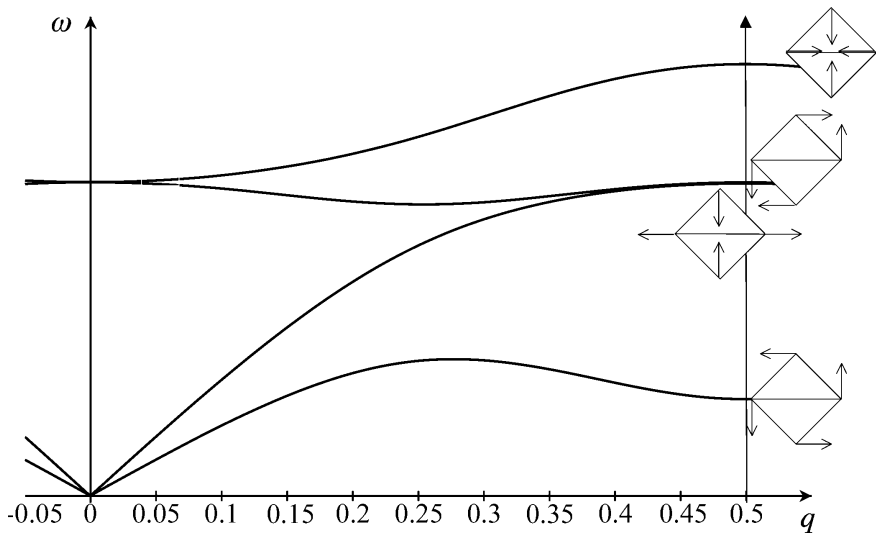

Figure 5

Phonon dispersion curves of a chessboard representing a single layer in $\mathrm{HgI}_{2}$, comprising only I atoms and in-plane atomic displacements. The wavevector in the $\Sigma$ direction is $\mathbf{q}=2 \pi q\left(\mathbf{a}_{1}^{*}+\mathbf{a}_{2}^{*}\right)$. Eigenvectors at point $M, q=\frac{1}{2}$. 
$\left.\frac{1}{2}\right)$. For this direction, the types of eigenvector are easily guessed to be either $e_{1},-e_{2}, e_{2},-e_{1}$ or $e_{1}, e_{2}, e_{2}, e_{1}$ (displacement amplitudes along $\mathbf{a}_{1}$ and $\mathbf{a}_{2}$ of atoms 1 and 2). In both cases, the dynamical matrix reduces to a $2 \times 2$ matrix, whose eigenvalues are

$$
\begin{aligned}
(m / 2) \omega_{1,2}^{2}= & \left(\alpha_{1}+\alpha_{2}\right)+\left(-\alpha_{1}+\alpha_{2}+\beta_{1}+\beta_{2}\right. \\
& \left.+\gamma_{1}+\gamma_{2}\right) \sin ^{2} \pi q \pm X \\
(m / 2) \omega_{3,4}^{2}= & \left(\alpha_{1}+\alpha_{2}\right)+\left(\alpha_{1}-\alpha_{2}+\beta_{1}+\beta_{2}\right. \\
& \left.+\gamma_{1}+\gamma_{2}\right) \sin ^{2} \pi q \pm X \\
X= & \left(\alpha_{1}+\alpha_{2}\right)^{2} \cos ^{4} \pi q \\
& \left.+\left(\beta_{1}-\beta_{2}-\gamma_{1}+\gamma_{2}\right)^{2} \sin ^{4} \pi q\right\}^{1 / 2},
\end{aligned}
$$

where $m$ is the mass of the atom. We now further simplify the model by assuming that all I-I interactions of the filled tetrahedra are equal and that atoms do not interact across the white squares: $\alpha_{1}=\beta_{1}, \alpha_{2}=\beta_{2}, \gamma_{1}=\gamma_{2}=0$. The rigidity of the white squares depends only on the transverse deformation force constant $\alpha_{2}$. The resulting phonon dispersion curves are shown in Fig. 5 for $\alpha_{1} / \alpha_{2}=20$. The lowest transverse acoustical mode, corresponding to the largest displacement amplitudes, is of particular interest. At the border point $M$ of the Brillouin zone, $q=\frac{1}{2}$, this curve shows a minimum with eigenvalue $\omega^{2}=$ $8 \alpha_{2} / m$. The eigenvector is the displacement pattern of Fig. 1 . The qualitative form of the phonon curves is similar for $\alpha_{1} / \alpha_{2}=$ 10 and does not change much as long as $\alpha_{2}$ is kept reasonably small. With increasing $\alpha_{2}$, the white squares become stiffer and the minimum at $q=\frac{1}{2}$ gradually disappears. For $\alpha_{1} \simeq \alpha_{2}$, the dispersion curves approach those of an uncolored square tiling (with no optical branches since the unit cell becomes smaller). In contrast, for $\alpha_{2}=0$ the frequency at $q=\frac{1}{2}$ is zero, and there should be a phase transition driven by a soft mode. Such a transition has not been observed. The vibration frequencies inside the Brillouin zone are larger than at point $M$ on the border, because these modes lead to deformations of the tetrahedra; the frequencies involve length deformation terms and are thus higher. This is also the case for a more complete model comprising $\mathrm{Hg}$.

4.2.2. Vibrations of an isolated $\mathbf{H g l}_{2}$ layer. The more complete model based on an isolated three-dimensional $\mathrm{HgI}_{2}$ layer with all first-neighbor $\mathrm{I}-\mathrm{I}$ and $\mathrm{Hg}-\mathrm{I}$ force-constant tensors can be solved explicitly for $q_{1}=q_{2}=\frac{1}{2}$ at point $M$ of the Brillouin zone. Most importantly, the complete layer possesses a librational mode whose frequency depends on a weak transverse force constant and interactions between different tetrahedra (across voids), and is identical to the soft mode of the chessboard model. Indeed, three modes of (3) and Fig. 5 also exist in the complete layer. Only the mode above the lowest of Fig. 5 also contains a movement of $\mathrm{Hg}$ along $+\mathbf{c}$ (for the arrows chosen in the figure). The frequencies of the other five modes (four of which are pairwise degenerate) all depend on longitudinal force constants and are thus large compared with the soft mode. Two of them mainly involve the I atoms, the other three mainly $\mathrm{Hg}$. The frequencies of the former are dominated by longitudinal I-I force constants, those of the latter by the $\mathrm{Hg}-\mathrm{I}$ force constants. The eigenvectors of the latter are close to displacements of $\mathrm{Hg}$ along $\mathbf{a}_{1}, \mathbf{a}_{2}$ and $\mathbf{c}$, i.e. perpendicular to the edges of the tetrahedra. If the $\mathrm{Hg}-\mathrm{I}$ force constants were smaller than the I-I force constants, modes dominated by the former would have low frequencies and the corresponding larger amplitudes would give an explanation of the surprisingly large displacement factors $\mathbf{U}(\mathrm{Hg})$.

4.2.3. Vibrations of the complete $\mathbf{H g I}_{2}$ structure. Finally, we discuss the soft libration mode of the complete $\mathrm{HgI}_{2}$ structure. The full $18 \times 18$ dynamical matrix for wavevectors $\left(q_{1}, q_{2}, 0\right)$ assuming nearest-neighbor interactions has been deposited. One type of $\mathrm{Hg}-\mathrm{I}$ interaction and four types of I-I interactions (apical and equatorial $\mathrm{HgI}_{4}$ edges, $\mathrm{I}-\mathrm{I}$ contacts in and between layers) are defined by 23 symmetry-independent force constants. For $q_{1}=q_{2}=\frac{1}{2}$ at point $M$, eight types of eigenvectors are obtained for the 18 modes, corresponding to two $3 \times 3$ and six $2 \times 2$ matrices to be diagonalized. It turns out that in a layer pair, the librational mode in one layer is coupled with the mode above the lowest of Fig. 5 in the other layer, as shown in Fig. 6 . The instantaneously deformed layers possess the same symmetry ( $p \overline{4} 2_{1} m$ with respect to the translations $\mathbf{a}_{1} \mp \mathbf{a}_{2}$ ): the librations deform the empty tetrahedra while in the adjacent layer the occupied tetrahedra are deformed. These deformations are coupled via the anisotropic component (term $\tau_{12}=\tau_{21}$ ) relative to the layer plane of the interlayer force constant tensor $\tau$. Without this anisotropic component $\left(\tau_{12}=0\right)$, a layer could execute librational movements while the adjacent layer remained static. The frequency of the librations depends not only on the force constants of an

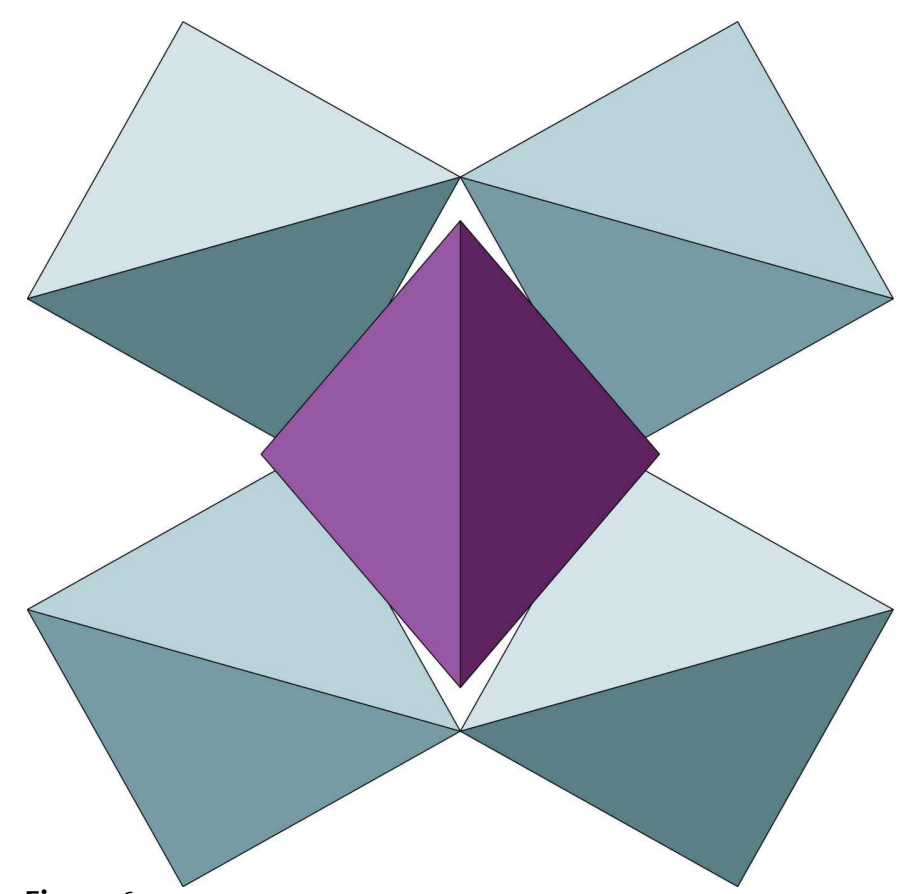

Figure 6

Qualitative instantaneous view of a pair of $\mathrm{HgI}_{2}$ layers deformed by the low-frequency mode of librating tetrahedra (program ATOMS; Dowty, 2006). If in one layer the tetrahedra librate, they are deformed in the adjacent layer. Alternatively, the mode may be represented by librations of (slightly) deformed tetrahedra. The displacements in different layers are then not coupled, while the frequency depends on the interlayer force constants. 
isolated layer, but also on the components $\tau_{11}$ and $\tau_{22}$ of the tensor $\tau$ which express the friction between layers. Evidently, there are two degenerate modes of this type, since all layers are symmetrically equivalent. The librational movement is expected to be considerably larger than the deformation of the $\mathrm{HgI}_{4}$ tetrahedra since the latter requires much higher energy (Fig. 5) and contributes to $U_{22}(\mathrm{I})$, which is the smallest of the observed displacement tensor components.

We conclude that our interpretation of the experimental results in terms of an important librational motion of the $\mathrm{HgI}_{4}$ tetrahedra (Fig. 1) is well supported by the phonon model. The simple chessboard model shows the salient features and gives similar results at point $M$ as a corresponding three-dimensional model. The thermal vibrations of a layer may be lumped into two contributions: the coupled librations of the tetrahedra creating the displacement pattern of Fig. 1 with wavevector $\mathbf{q}=\pi\left(\mathbf{a}_{1}^{*}+\mathbf{a}_{2}^{*}\right)$ and translations of rigid layers close to $\mathbf{q}=0$. Optical modes at $\mathbf{q}=0$ are pairwise degenerate (Prevot et al., 1978; Biellmann \& Prevot, 1980) and are expected to contribute equally to $U_{11}(\mathrm{I})$ and $U_{22}(\mathrm{I})$; they cannot explain the observed $U_{11}(\mathrm{I})>>U_{22}(\mathrm{I})$. In addition, the large displacement parameters of $\mathrm{Hg}$ seem to indicate that the $\mathrm{Hg}-\mathrm{I}$ force constants may be considerably smaller than the I-I force constants.

\subsection{Mechanism of anharmonic motions}

In this section we demonstrate that the libration mode generates additional anharmonic effects. The thermal motions of rigid molecules are described by TLS theory (Schomaker \& Trueblood, 1968). For an isolated rigid tetrahedron with the symmetry $\overline{4} m 2$, the independent terms are $L_{11}=L_{22}, L_{33}$ (libration tensor); $T_{11}=T_{22}, T_{33}$ (translation tensor); $S_{12}=S_{21}$ (correlation tensor); all other terms being 0 ( $\overline{4}$ plus $m m 2$ in Willis \& Pryor, 1975). $L_{11}$ and $S_{12}$ contribute to $U_{23}(\mathrm{I})$. This shows that the corner-linked I-tetrahedra in $\mathrm{HgI}_{2}$ cannot move as rigid bodies: the space-group symmetry imposes $U_{23}(\mathrm{I})=0$ and therefore $L_{11}=S_{12}=0$. In other words, the I atoms cannot move on curved trajectories as would be the case for a librating free molecule. The mean-square libration amplitude $L_{33}$ is easily calculated as (Willis \& Pryor, 1975; Schomaker \& Trueblood, 1968)

$$
L_{33}=4 a^{-2} \Delta U\left[\mathrm{rad}^{2}\right]
$$

with $\Delta U=\left[U_{11}(\mathrm{I})-U_{22}(\mathrm{I})\right]$, while the translation terms are $T_{11}=T_{22}=U_{22}(\mathrm{I})$ and $T_{33}=U_{33}(\mathrm{I})$. Table 2 shows the r.m.s. libration amplitudes $\left(L_{33}\right)^{1 / 2}$ in degrees.

During the libration described by $L_{33}$, the tetahedron undergoes an anharmonic deformation of bond lengths whose frequency is twice the harmonic libration frequency: the deformation due to $U_{11}(\mathrm{I})$ is the same for instantaneous displacements of I along $+\mathbf{a}_{1}$ and along $-\mathbf{a}_{1}$. This distortion of the tetrahedron may be estimated as follows. Suppose that the I atom at the equilibrium position $\frac{1}{4}, \frac{1}{4}, z_{0}(\mathrm{I})$, which belongs to the tetrahedron of the $\mathrm{Hg}$ atom at $\frac{1}{4}, \frac{3}{4}, \frac{1}{4}$, is instantaneously displaced by $\delta a(\AA)$ along the $+\mathbf{a}_{1}$ cell axis, and by $\varepsilon c(\AA)$ along the $+\mathbf{c}$ axis. The instantaneous $\mathrm{Hg}-\mathrm{I}$ bond length becomes

$$
\begin{aligned}
d= & {\left[d_{0}^{2}+a^{2} \delta^{2}+2 \epsilon c^{2}\left(z_{0}-\frac{1}{4}\right)+c^{2} \epsilon^{2}\right]^{1 / 2} } \\
& \simeq d_{0}+(a \delta)^{2} / 2 d_{0}+\left[\epsilon c^{2}\left(z_{0}-\frac{1}{4}\right)\right] / d_{0},
\end{aligned}
$$

where $d_{0}$ is the equilibrium distance. Analogous equations exist for the I-I contacts. The harmonic in-plane $(\delta)$ and outof-plane $(\varepsilon)$ movements are uncorrelated. However, the second-order term $\delta^{2}$ is correlated with $\varepsilon$ through the thirdorder anharmonic Gram-Charlier term $U_{113}$, which is allowed by the point-group symmetry $m m 2$ of I. Since our experimental data gave no information on the third-order anharmonicity of I, we consider different assumptions:

(i) The $\mathrm{Hg}-\mathrm{I}$ distance does not change during the vibration, i.e. $d=d_{0}$ at all times. Since the average value $\left\langle a^{2} \delta^{2}\right\rangle=$ $\Delta U$, the average value of $\varepsilon$ becomes $<\varepsilon>=-\Delta U /\left[2 c^{2}\left(z_{0}-\frac{1}{4}\right)\right]$. The observed $z$ coordinate is $z(\mathrm{I})=z_{0}+<\varepsilon>$ and the librationcorrected equilibrium $\mathrm{Hg}-\mathrm{I}$ distance is $d_{0} \simeq d_{\text {obs }}+\Delta U / 2 d_{\text {obs. }}$. This correction never exceeds 2 s.u. The tetrahedron vibrates with deformations of the $\mathrm{I}-\mathrm{Hg}-\mathrm{I}$ angles, i.e. deformations of the I-I contacts. The assumption of a rigid bond length is, however, not very convincing in view of the large thermal displacements of $\mathrm{Hg}$. An alternative assumption is:

(ii) $\varepsilon=0$ at all displacements $\delta$, i.e. the libration is not coupled with an out-of-plane movement of I. We may assume that the probability of finding a displacement $a \delta$ is given by a Gaussian distribution $G(a \delta)$ with variance $\Delta U, G(a \delta)=$ $(2 \pi \Delta U)^{-1 / 2} \exp \left[-(a \delta)^{2} / 2 \Delta U\right]$. The average distance is easily calculated by integrating $d G(a \delta)$ from $\delta=-\infty$ to $\infty$ : $\langle d\rangle=d_{0}+$ $\Delta U / 2 d_{0}$. In opposition to model (i), this is not a libration correction, but a dynamic average. The equilibrium position of I at $\delta=0$ is not affected by the libration. The probability density function (p.d.f.) of $d$ is obtained by substituting $a \delta=$ $2 d_{0}\left(d-d_{0}\right)$ into the Gaussian p.d.f. $G(a \delta)$. By integrating the result from $d_{0}$ to $d$, the cumulative distribution function $C(d)$ is obtained, which gives the probability for finding a bond length smaller than $d$

$$
\begin{aligned}
C(d) & =\operatorname{erf}\left[a \delta(2 \Delta U)^{-1 / 2}\right]=\operatorname{erf}\left[\left(d_{0}\left(d-d_{0}\right) / \Delta U\right)^{1 / 2}\right] \\
\operatorname{erf}(x) & =2(\pi)^{-1 / 2} \int_{0}^{x} \exp \left(-t^{2}\right) \mathrm{d} t
\end{aligned}
$$

$100(1-C)$ gives the percentage of bonds longer than $d$. At $293 \mathrm{~K}, 2.3 \%$ of the bonds are extended by more than $0.01 d_{0}=$ $0.028 \AA=6$ s.u. of $d_{0}$ (Table 2). At $200 \mathrm{~K}, 4.6 \%$ of the bonds are lengthened by more than $0.005 d_{0}=9$ s.u. At $100 \mathrm{~K}$, the values are $4.7 \%$ for $0.002 d_{0}=5$ s.u. and at $60 \mathrm{~K} 1.1 \%$ for $0.002 d_{0}=6 \mathrm{~s}$.u. These values may not seem to be impressive, but the proposed bond extensions are statistically significant and the percentages of longer bonds are not negligible. On the level of 2 s.u., the percentage of longer bonds is $16 \%$ at $100 \mathrm{~K}$, and increases to $37 \%$ at 200 and $293 \mathrm{~K}$. The model is easily generalized to produce longer dynamical bond extensions: the I atoms may be allowed to move along $+\mathbf{c}$ in the opposite direction of model (i), i.e. $\varepsilon=k \delta$ positive and proportional to the displacement $\delta$. Since $\varepsilon$ contributes linearly to the distance, 
$k$ values as small as 0.05 or 0.1 have a considerable effect on the bond lengthening.

Assumption (ii) suggests an explanation of the large movements of $\mathrm{Hg}$ and the proposed small $\mathrm{Hg}-\mathrm{I}$ force constants. The dynamic lengthening of the $\mathrm{Hg}-\mathrm{I}$ bonds permits $\mathrm{Hg}$ to vibrate with larger amplitudes than the closepacked I atoms, and the single-atom potential is indeed expected in this model to soften with the lengthening of the bonds. It is known that the color of $\mathrm{HgI}_{2}$ fades with decreasing temperatures, being orange at $80 \mathrm{~K}$. This suggests that the red color is associated with the lengthened $\mathrm{Hg}-\mathrm{I}$ bonds and disappears when bond lengths remain close to $d_{0}$. The color change has been taken as indicative of a phase transition at low temperatures. However, our present explanation does not require the occurrence of a phase transition, for which we find no evidence in the present work.

\section{Conclusions}

The dominant features of the thermal mean-square displacements in $\mathrm{HgI}_{2}$ as functions of temperature are:

(i) the $U(\mathrm{I})$ tensor is very anisotropic with $U_{11}(\mathrm{I})$ large,

(ii) the isotropic average $U_{\text {eq }}(\mathrm{Hg})>U_{\text {eq }}(\mathrm{I})$, and

(iii) the thermal motions of both atoms, except $U_{22}(\mathrm{I})$, are clearly anharmonic and indicate a softening of the isolatedatom potential at large amplitudes.

Observation (i) is explained by a low-frequency soft phonon mode at the surface point $M$ of the Brillouin zone, wavevector $\mathbf{q}=\pi\left(\mathbf{a}_{1}^{*}+\mathbf{a}_{2}^{*}\right)$ involving cooperative librations of the $\mathrm{HgI}_{4}$ tetrahedra; (ii) suggests relatively small force constants of the $\mathrm{Hg}-\mathrm{I}$ bond and, correspondingly, modes allowing $\mathrm{Hg}$ to move quite freely inside the tetrahedra without being much affected by longitudinal I-I force constants. We propose for (iii) a mechanism where the librations of the $\mathrm{HgI}_{4}$ tetrahedra impose a dynamic vibration of all the bonds, and in particular of the $\mathrm{Hg}$ - I bond: the bond lengthens with respect to the equilibrium length $d_{0}$ in phase with, but with twice the frequency of the libration, with $d_{0}$ not being an average but the shortest bond length. The isolated atom potential of $\mathrm{Hg}$ thereby becomes anharmonic and admits r.m.s. vibration amplitudes of $\mathrm{Hg}$ which are considerably larger than those of I, and increase faster with temperature. This model also suggests a qualitative explanation for the lightening of the color of $\mathrm{HgI}_{2}$ from red to orange at $c a 80 \mathrm{~K}$. However, there is no evidence for the IIIIV phase transition. It is not known whether the color change has a well defined onset and might thus indicate a phase boundary.

While more accurate measurements of the thermal displacements in $\mathrm{HgI}_{2}$ by single-crystal synchrotron X-ray and neutron diffraction would be welcome, a confirmation of our model requires a determination of the phonon dispersion curves in the $\Sigma$ direction, and particularly at point $M$, by inelastic neutron scattering. The force constant model adopted by Sim et al. (1994) disregards I-I interactions of four of the six $\mathrm{HgI}_{4}$ tetrahedral edges and is insufficient for a calculation of these curves.

This work is based on experiments performed at the Swiss spallation neutron source SINQ, Paul Scherrer Institute, Villigen, Switzerland. The authors thank the SINQ committee for the provided neutron beam-time and the Swiss National Fund for financial support. H. B. thanks the Danish Research Councils for additional financial support. We are indepted to Dr V. Pomjakushin (Paul Scherrer Institute) for help with part of the measurements and to Dr K. Schenk (Ecole Polytechnique Fédérale, Lausanne) for help with figures and CIFs.

\section{References}

Biellmann, J. \& Prevot, B. (1980). Infrared Phys. 20, 99-106.

Bube, R. H. (1957). Phys. Rev. 106, 703-715.

Bürgi, H. B., Capelli, S. C. \& Birkedal, H. (2000). Acta Cryst. A56, 425-435.

Dowty, E. (2006). ATOMS, Version 6.3. Shape Software, 521 Hidden Valley Road, Kingsport, Tennessee, USA; http://www.shapesoftware.com.

Fischer, P., Frey, G., Koch, M., Könnicke, M., Pomjakushin, V., Schefer, J., Thut, R., Schlumpf, N., Bürge, R., Greuter, U., Bondt, S. \& Berruyer, E. (2000). Physica B, 276-278, 146-147.

Fischer, W. E. (1997). Physica B, 234-236, 1202-1208.

Gospodinov, M. M., Petrova, D., Yanchev, I. Y., Daviti, M., Manolopoulou, M., Paraskevopoulos, K. M., Anagnostopoulos, A. N. \& Polychroniadis, E. K. (2005). J. Alloys Compd. 400, 249251.

Hostettler, M. (2002). PhD thesis, Faculté des Sciences, Université de Lausanne, Switzerland.

Hostettler, M., Birkedal, H. \& Schwarzenbach, D. (2001). Chimia, 55, 541-545.

Hostettler, M., Birkedal, H. \& Schwarzenbach, D. (2003). Helv. Chim. Acta, 86, 1410-1422.

Hostettler, M. \& Schwarzenbach, D. (2005). C. R. Chim. 8, 147-156. Jeffrey, G. A. \& Vlasse, M. (1967). Inorg. Chem. 6, 396-399.

Karmakar, S., Deb, S. K. \& Sharma, S. M. (2005). Physica B, 369, $287-$ 292.

Lenstra, A. T. H., Van Loock, J. F. J., Rousseau, B. \& Maes, S. T. (2001). Acta Cryst. A57, 629-641.

Prevot, B., Schwab, C. \& Dorner, B. (1978). Phys. Status Solidi (b), 88, 327-333.

Rodriguez-Carvajal, J. (1993). Physica B, 192, 55-69.

Rousseau, B., Maes, S. T. \& Lenstra, A. T. H. (2000). Acta Cryst. A56, 300-307.

Schomaker, V. \& Trueblood, K. N. (1968). Acta Cryst. B24, 63-76.

Sim, H.-K., Chang, Y.-C. \& James, R. B. (1994). Phys. Rev. B, 49, 4559-4563.

Tonkov, E. D. \& Tikhomirova, N. A. (1971). Sov. Phys. Cryst. 15, $945-$ 946.

Willis, B. T. M. \& Pryor, A. W. (1975). Thermal Vibrations in Crystallography, pp. 175-185. London: Cambridge University Press. 